\title{
Making the case for a "CERN for analogs"
}

\author{
Alfredo lorio \\ Institute of Particle and Nuclear Physics \\ Faculty of Mathematics and Physics, Charles University \\ V Holešovičkách 2, 18000 Praha 8, Czech Republic \\ E-mail: alfredo.iorio@mff.cuni.cz
}

\begin{abstract}
Scientific and practical arguments are offered in favor of a facility where to explore, with analogs, the otherwise unreachable territories of the theoretical investigations on the fundamental components of nature. In this facility, theorists, both of the high-energy and of the condensed matter types, should work next to experimentalists, mostly of the condensed-matter type, and technologists. I call this facility HELIOS, an evocative name for something that should shed light on the darkness of the unknown, and an acronym for High Energy Laboratory for Indirect ObservationS.
\end{abstract}

40th International Conference on High Energy physics - ICHEP2020

July 28 - August 6, 2020

Prague, Czech Republic (virtual meeting) 


\section{Analogs}

When I am asked to explain what quantum mechanics (QM) is about, I usually hit the ground with a foot. That solid floor under my feet could not exist, if QM were not true. No matter the substance, the electrons would all collapse on the nucleus, and would not make the bonds that hold together the structure of the solid. In a way, a solid, as large as a mountain, is a molecule, as large as a mountain. And a molecule can only exist because QM is true. Therefore, the larger the mountain, the more evident its quantum nature. The above is indisputable, and it was clear already to Schrödinger [1]. Nonetheless, the standard folklore is that QM is what works only in the extremely small, where mysterious things happen, and those mysteries cannot be perceived at our scales. According to that folklore, that stuff is out of reach for us, poor and clumsy macroscopic beings. Well, that is not true. We can reach that exotic realm, simply because we are immersed into it! We need to know what to look for, and that is not easy, but neither impossible. Similarly (or analogously, would be perhaps a more appropriate word in this paper), we should be able to reach, here and now, the ultimate building blocks (were they strings, loops, superparticles, dark matter, or whatever), no matter how far they might be, in the small, in the large, or in the past. Their direct or indirect effects, on the world as it is here and now, must be tangible. That is the rationale behind testing fundamental theories with analogs.

It was Feynman who argued that different systems obey the same equations (of electrostatics, in that lecture [2]) when there is one structure common to all: "What is common to all our problems is that they involve space and that we have imitated what is actually a complicated phenomenon by a simple differential equation. [...] Are they [the electrostatic equations, ed] also correct only as a smoothed-out imitation of a really much more complicated microscopic world? Could it be that the real world consists of little Xons which can be seen only at very tiny distances? And that in our measurements we are always observing on such a large scale that we cant see these little Xons, and that is why we get the differential equations?" [2] These days we have refined our art of imitating one system with another. Many analog models are available, and beautiful experiments are performed, especially for the Hawking radiation. All of this taught us that, when one sets-up a proper analog system, e.g., of a black hole, then it can get from there first hand information on... the black hole! So why put one's life ${ }^{1}$ in danger traveling till the nearest black hole? Why use an enormous amount of resources to try to reach those energies with particle accelerators, when the tip of a pencil [3] might serve the scope decently well? Of course, probes like gravitational waves, or cosmic rays, are precious, but why not to make the most of the indirect evidences that analog systems might offer? The reason why this is not happening yet, at the least not at the scale it should be happening, is that the relation between different systems, that we call analogy, still needs a universally accepted definition. The relation between different systems that we call symmetry indeed enjoys rigorous and universally accepted definitions, both from the mathematical and the physical points of view. None doubts that the use of such relation is legitimate: I test system $A, A$ is symmetric to $B$, I have tested system $B$. These systems can look very different, but once we have established the symmetry, we know that it is one and the same system. These days, due to the

\footnotetext{
${ }^{1}$ Actually, many more than one life would be necessary to reach there... The fastest man-made spacecraft, Voyager 1 , travels at about $c / 18000$, so it would reach the nearest black hole, that is about 1000 light years away, in about 18 million years.
} 
increasing interest, philosophers are intensively working on those issues, see, e.g. [4]. Our point of view, discussed in [5], is that all we need is: i) to rigorously identify the structure/aspect/feature common to both systems, and once that is done, ii) we have to find the mathematical relations between the two systems, that indeed make them one.

A beautiful example are the supernova explosions simulated in the laboratory by plasma implosions induced by intense lasers, a field of experimental physics to which laboratories like the National Ignition Facility at the Lawrence Livermore Laboratory, U.S.A., and the Laser MegaJoule in Bordeaux, France, have devoted considerable efforts. The Euler equations of fluid dynamics are invariant under an inversion transformation [6]

$$
\mathscr{I}: t \rightarrow-1 / t \quad \vec{x} \rightarrow \vec{x} / t
$$

that is an arbitrary uniform expansion or contraction of the system. This symmetry is studied in cosmology, and allows to map an explosion problem to a dual implosion problem. In principle this duality allows the complete three-dimensional evolution of highly structured explosion ejecta to be modeled using a static target in an implosion facility. From (1.1) it is clear that such a symmetry group is larger than the Galilei group, and in [7] the maximal invariance group was determined to be the semi-direct product for the Galilei group with $\operatorname{SL}(2, R)$, the latter containing time-translations, dilations, and the inversion $\mathscr{I}$. Those results had an important impact on the field.

In the days of the AdS/CFT correspondence [8], relating gravity and matter, of quantum gravity models inspired by condensed matter [9], like crystalline gravity, (see also [10]), and of the great advancement of analog experiments [11] (see also the review [12]), we believe that the times are mature for a dedicated laboratory, entirely devoted to test fundamental theories with analogs [13]. The other side of the story is that analogs are often important materials for technological applications, like graphene [14]. Henceforth, such a place would be an invaluable think-tank where unconventional thinking would be routinely applied, to create new technology and to solve fundamental problems. With our group in Prague, we have moved some steps in that direction focusing on Dirac materials, and have explored various theoretical [15] and experimental [16] directions. Many other groups around the world are taking similar roads, with different systems [11] (see also the review [12]) and with thrilling success. What is missing is a lab built with the same spirit of CERN, that unifies, systematizes and organizes those efforts, but also raises the status of this research to a quest to reach beyond the known. Let me call this facility High Energy Lab for Indirect ObservationS[13] (HELIOS).

\section{Goals of HELIOS}

Test fundamental theories: The prominent goal of HELIOS is to solve, with analog experiments, key open problems of the high energy theoretical investigation. First, the community should identify a list of key open problems that HELIOS will be able to attack. Such list should include those problems whose solution cannot be found solely with the theoretical analysis, as different competing theories give different answers. Furthermore, they must be problems that can clearly be faced via a reliable analog. This latter point is the most crucial. The first example that comes to the forefront is the information paradox, that might or might not take place during black hole 
evaporation, see, e.g., [17]. On the one hand, the ultimate answer can only come from an experiment. On the other hand, it is a problem that analog must be able to handle. Give experimental perspectives of the abstract research: HELIOS can also provide an experimental playground to any theoretical scenario. This is not as testing the given theory, but rather to find aspects of that investigation that can have an experimental facet. Thus, black hole physicists could build and play with acoustic quasinormal modes, whereas string theorists could learn about Liouville field theory constructing membranes of constant Gaussian curvature, etc. I find this part of HELIOS's mission of paramount epistemological and educational importance. It has been a peculiarity of these decades that the most theoretical investigation completely decoupled from experiments. The two carriers, for theorists and experimentalists, are completely separated. This is clearly a mistake, and HELIOS could serve also the scope of solving this epistemological vulnus. In fact, aspects of any theoretical construction can be reproduced in analogs, including SUSY, string theory, loop quantum gravity, extra dimensions, Generalized Uncertainty Principles, and all the hep-th bestiary. "Find the Xons": HELIOS should then have the grand goal of putting on firm basis the whole analog approach to physics. In other words, it should try to seriously investigate the questions posed by Feynman, and previously discussed in this paper. We may say that this part of its mission is to "find the Xons". High intensity cross-fertilization: Technological byproducts are listed as goals of any fundamental research facility, including CERN that lists among the goals of its research[18] "Advancing the frontiers of technology". Having often to deal with novel materials, already under intense scrutiny for technological applications, HELIOS has a much clearer vocation for innovative technology. The case of graphene, a bi-dimensional nanomaterial, with an enormous interest for industrial applications, is paradigmatic. In a way, we can say that, while for CERN's priorities technologists are a step below fundamental physicists, in HELIOS the two categories would be much closer. Hence, in HELIOS the cross-fertilization will be highly effective and intense: theoretical problems might be solved by technologists, technology problems might be solved by fundamental physicists. Costs: This is not a study of the feasibility of HELIOS, not even a preliminary one, but rather a place were we offer scientific and technological arguments in favor of the construction of this facility. Nonetheless, on a semi-serious note, let me try to quantify very approximately the costs of such an enterprize, in euros. I focus on what will be necessary for the experiments with graphene, and then will brutally multiply by ten to include into one facility a good sample of the analogs currently at our disposal [12]. For the "graphene module" of HELIOS, first one should be able to fabricate nanostructures of any wanted geometry, with the highest precision. Existing machines, e.g. the ORION NanoFab Helium Ion Microscope, cost around 0.8 million, but are still not what necessary for HELIOS. Therefore, a dedicated machine of this kind should be built. I judge this to cost 1.5 million, or more. Another important component of this module is a laser, to mimic a gravitational interaction. To have very short and well controlled pulses, necessary to probe the bidimensional material, we can estimate 0.5 million, and including the diagnostics, a total of 1 million. Of course, one needs also all sort of measuring devices, in particular STM machines are the most appropriate for certain experiments [3], and, including microscopes, for all these instruments I estimate at least 2.5 million. We would like HELIOS to be self-sufficient, hence it should also be able to produce graphene, e.g., via chemical vapor deposition (CVD), or improved methods. For this, and other means of production, I estimate a cost of 1 million. All together, this amounts to 6 million. It is extremely important that HELIOS has its own powerful computa- 
tional cluster, especially to carry on the necessary simulations, before embarking for any kind of experiment. This cluster will serve all the different analog modules, and its cost should be around 5 million. Furthermore, dedicated buildings are crucial, to merge theorists, experimentalists and technologists in one common environment. To host machines, instrumentation and personnel, and to include conference halls and all sort of open spaces for the dissemination of knowledge, and for the constant interaction with industry, such a structure will not cost less than 20 million. Finally, and most importantly, the researchers and technicians will have to be paid according to international standards, to guarantee the highest motivation and attract the best. Considering 8 people per module, at 100,000 per person per year, and including the personnel of the administration, security etc, this chapter amounts to 10 million per year, at full regime.

Summing up, to take off HELIOS would need: $(6 \times 10)+5+20+10$ million, that is roughly 0.1 billion. CERN budget for 2020 is about 1.1 billion, see [19]. While it is still slightly less than the 2019/20 budget of Barcelona football club, HELIOS would take off with only a fraction of that. Having listed above what we might earn with HELIOS, the cost-benefit comparison of the two facilities appears to me to be in great favor of HELIOS...

\section{Conclusions}

In HELIOS we shall be able to explore, with analogs, the otherwise unreachable territories of the theoretical landscape. On the other hand, the technological spin-offs are potentially many more than for other facilities with similar goals. In particular, the unconventional use of advanced materials to reproduce exotic scenarios will push ahead the limits of technology, and may furnish unsuspected solutions to practical problems.

\section{Acknowledgements}

I am indebted to Raffaele G. Agostino, Xinliang Feng, and Tadzio Levato for helping me figure out the costs. I thank Antonello Scardicchio for hosting me while thinking about these matters, and ICTP for inspiration. The grant Charles University Research Center (UNCE/SCI/013) is gladly acknowledged.

\section{References}

[1] E. Schrödinger, What is Life? Cambridge Univ. Press, Cambridge, UK. 1944.

[2] R. Feynman, R. Leighton, M. Sands and M. Gottlieb, The Feynman Lectures on Physics, Pearson/Addison-Wesley, Boston 2006 (Electrostatic analogs, Vol II, Chp 12).

[3] A. Iorio and G. Lambiase, The Hawking-Unruh phenomenn on graphene, Phys. Lett. B 716 (2012) 334; Quantum field theory in curved graphene spacetimes, Lobachevsky geometry, Weyl symmetry, Hawking effect, and all that, Phys. Rev. D 90 (2014) 025006.

[4] R. Dardashti, K. P. Y. Thébault, E. Winsberg, Confirmation via analogue simulation: What dumb holes could tell us about gravity, Brit. J. Phil. Sci. 68 (2017) 55; K. Crowther, N.S. Linnemann, and C. Wüthrich, What we cannot learn from analogue experiments, Synthese (2019) https://doi.org/10.1007/s11229-019-02190-0 
[5] A. Iorio, Two arguments for more fundamental building blocks, J Phys: Conf. Ser. 1275 (2019) 012013.

[6] L. O’ C Drury and J. T. Mendonça, Phys. Plasmas 7 (2000) 5148.

[7] L. O'Raifeartaigh, V. V. Sreedhar, The maximal kinematical invariance group of fluid dynamics and explosion-implosion duality, Ann. Phys. 293 (2001) 215-227.

[8] J. Maldacena, The large-N limit of superconformal field theories and supergravity, Int. J. Theor. Phys. 38 (1999) 1113; The illusion of gravity, Sci. Am. April 1, 2007

[9] H. Kleinert, Multivalued Fields in Condensed Matter, Electrodynamics, and Gravitation, World Scientific, Singapore 2008; G. 't Hooft, Crystalline Gravity, Int. J. Mod. Physics A 24 (2009) 3243 3255; R. Loll, Discrete approaches to quantum gravity in four dimensions, Liv. Rev. Rel. 1 (1998) 13.

[10] G. Acquaviva, A. Iorio, M. Scholtz, On the implications of the Bekenstein bound for black hole evaporation, Ann. Phys. 387 (2017) 317; G. Acquaviva, A. Iorio, L. Smaldone, Bekenstein bound from Pauli principle, Phys. Rev. D 102 (2020) 106002.

[11] J.R. Muñoz de Nova, K. Golubkov, V.I. Kolobov, J. Steinhauer, Observation of thermal Hawking radiation and its temperature in an analogue black hole, Nature 569 (2019) 688; J. Gooth, et al, Experimental signatures of the mixed axial-gravitational anomaly in the Weyl semimetal NbP, Nature 547 (2017) 324; J. Drori, Y. Rosenberg, D. Bermudez, Y. Silberberg, U. Leonhardt, Observation of Stimulated Hawking Radiation in an Optical Analogue, Phys. Rev. Lett. 122 (2019) 010404.

[12] C. Barceló, S. Liberati, M. Visser, Analogue Gravity, Liv. Rev. Rel. 14 (2011) 3.

[13] A. Iorio, Novel materials to reach the unreachable, Fron. Mat. 1 (2015) 36; What after CERN? Opportunities from co-responding systems, J. Phys.: Conf. Ser. 626 (2015) 012035; HELIOS or the need for a CERN for analogs, lecture at the Workshop "Simulating gravitation and cosmology in condensed matter and optical system" 22-25 Jul 2019, ECTstar, Trento.

[14] A. Iorio, Weyl-gauge symmetry of graphene, Ann. Phys. 326 (2011) 1334; Curved spacetimes and curved graphene: a status report of the Weyl symmetry approach, Int. J. Mod. Phys. D 24 (2015) 1530013.

[15] A. Iorio, P. Pais, Revisiting the gauge fields of strained graphene, Phys. Rev. D 92 (2015) 125005; A. Iorio, P. Pais, (Anti-) de Sitter, Poincaré, Super symmetries, and the two Dirac points of graphene, Ann. Phys. 398 (2018) 265; A. Iorio, P. Kus, Vortex solutions of Liouville equation and quasi spherical surfaces, Int. J. Geom. Meth. Mod. Phys. 17 (2020) 2050106 [arXiv:2003.10902]; M. Ciappina, A. Iorio, P. Pais, A. Zampeli, Torsion in quantum field theory through time-loops on Dirac materials, Phys. Rev. D 101 (2020) 036021; A. Iorio, P. Pais, I.A. Elmashad, A.F. Ali, M. Faizal, L.I. Abou-Salem, Generalized Dirac structure beyond the linear regime in graphene, Int. J. Mod. Phys. D 27 (2018) 1850080; A.Iorio, G.Lambiase, P.Pais, F.Scardigli, Generalized uncertainty principle in three-dimensional gravity and the BTZ black hole, Phys. Rev. D 101 (2020) 105002 [arXiv:1910.09019].

[16] A. Iorio, Graphene: QFT in curved spacetimes close to experiments, J. Phys.: Conf. Ser. 442 (2013) 012056; S. Taioli, R. Gabbrielli, S. Simonucci, N.M. Pugno, A. Iorio, Lobachevsky crystallography made real through carbon pseudospheres, J. Phys.: Cond. Matt. 28 (2016) 13LT01.

[17] L. Susskind, Black Hole War: my battle with Stephen Hawking to make the world safe for quantum mechanics, Little, Brown and C., New York, USA 2008.

[18] https://home.cern/about/who-we-are/our-mission

[19] CERN Quick Facts 2020, CERN-Brochure-2020-006-Eng, from CERN website. 